DOI: https://doi.org/10.46296/gt.v5i9edespmar.0053

\title{
LA DINÁMICA FAMILIAR FRENTE AL DUELO POR LA PÉRDIDA DE UN MIEMBRO DEL SISTEMA
}

\section{FAMILY DYNAMICS IN THE FACE OF DUE FOR THE LOSS OF A MEMBER OF THE SYSTEM}

\author{
Ponce-Reyes Joselyn Viviana ${ }^{1}$; Rodríguez-Álava Leonor Alexandra ${ }^{2}$ \\ 1 Instituto de Posgrado, Facultad de Ciencias Humanísticas y Sociales, Universidad Técnica de \\ Manabí. Portoviejo, Ecuador. Correo: joovpr1996@gmail.com. \\ ORCID ID: https://orcid.org/0000-0003-0763-0956 \\ ${ }^{2}$ Instituto de Posgrado, Facultad de Ciencias Humanísticas y Sociales, Universidad Técnica de \\ Manabí. Portoviejo, Ecuador. Correo: leonor.rodriguez@utm.edu.ec. \\ ORCID ID: https://orcid.org/0000-0002-3034-1311
}

\begin{abstract}
Resumen
La dinámica familiar frente al duelo por la pérdida de un miembro del sistema es objeto de análisis debido a que se genera malestar y sus complicaciones inciden en las relaciones, funciones, y dinámica en general de la familia. Esta artículo es de revisión bibliográfica, por lo cual, tiene una metodología de tipo documental y bibliográfica porque permite recopilar información de fuentes confiables de investigaciones realizadas en diferentes lugares, que esclarecen el enlace entre estas variables, además aportan datos significativos sobre los cambios que sufre la familia en sus relaciones, funciones, roles y dinámica ante el duelo por fallecimiento de uno de sus miembros generan interés entre los estudiosos del tema. El objetivo del artículo es caracterizar los cambios en la dinámica familiar frente al duelo por un miembro del sistema. Entre los resultados que destacan se encuentra una relación entre los duelos complicados y la homeostasis de la dinámica familiar. La dinámica familiar presenta cambios antes las pérdidas de su estructura, y cuando estas carecen de capacidad de reorganización terminan en duelos complicados.
\end{abstract}

Palabras clave: Dinámica familiar, duelo, muerte, sistema.

\begin{abstract}
The family dynamics in the face of mourning for the loss of a member of the system is the object of analysis due to the fact that discomfort is generated and its complications affect the relationships, functions, and dynamics in general of the family. This article is a bibliographical review, therefore, it has a documentary and bibliographical methodology because it allows to collect information from reliable sources of investigations carried out in different places, which clarify the link between these variables, in addition to providing significant data on the changes that it undergoes. the family in its relationships, functions, roles and dynamics in the face of grief due to the death of one of its members generate interest among scholars on the subject. The objective of the article is to characterize the changes in family dynamics in the face of mourning for a member of the system. Among the results that stand out is a relationship between complicated duels and homeostasis of family dynamics. The family dynamics changes before the loss of its structure, and when they lack reorganization capacity, they end up in complicated duels.
\end{abstract}

Keywords: Family dynamics, duel, death, system.

Información del manuscrito:

Fecha de recepción: 06 de enero de 2022.

Fecha de aceptación: 24 de febrero de 2022.

Fecha de publicación: 04 de marzo de 2022. 


\section{INTRODUCCIÓN}

Ante la pérdida de un ser querido, se produce el duelo, considerado como un proceso normal que presenta características de declive en un período que llega a la fase de aceptación; sin embargo, existen también sistemas familiares que inciden en agravar la sintomatología propia del duelo, adoptando posturas complicadas que pueden afectar en mayor o menor medida la dinámica del sistema familiar.

Cely, Martínez \& Mcleod (2016) en su estudio realizado en Bogotá para analizar las transformaciones en la dinámica familiar por el deceso de un miembro de la familia considerando el ciclo vital de la misma, determinó que el factor influyente de las pérdidas en el cambio de las dinámicas de la familia está en relación al rol desempeñado por la persona fallecida, que es transferido a otro integrante. Es importante mencionar que, durante el cambio de roles, estas reestructuran sus actividades, por ende, su dinámica ante la pérdida de un ser querido.

En un estudio realizado en Costa Rica por Vargas, R. (2002) sobre duelo y pérdida, expresa que la intervención psicológica en duelo es un proceso complejo, el cual implica dejar a un lado vínculos con personas significativas, lo cual crea una conciencia de realidad que invita a enfrentar la pérdida, momento en que la persona comienza su proceso individual de duelo. Es innegable que toda persona que vive una pérdida afectiva debe transitar por un proceso, en este, se sentirá triste ante el hecho, debido a la connotación de la realidad, porque pierde un objeto del mundo externo y sabe cuál es, así la persona comienza con conductas anormales, no necesariamente patológicas que usualmente logra superar con el paso del tiempo.

En el contexto ecuatoriano, Martínez \& Santos (2020) en su estudio sobre el duelo familiar y el desarrollo emocional en adolescentes, demuestran que el proceso de duelo resulta en aprendizaje transcendental, debido a que la experiencia dolorosa da lugar a un autoconocimiento, el cual proporciona enseñanzas útiles para el desarrollo de la vida. Tras la pérdida se utilizan estrategias que permiten restaurar la vida hacia etapas de mayor satisfacción y 
espacios más edificantes en el proceso de vida.

En relación con las estrategias, se destaca la experiencia con adultos mayores no institucionalizados del centro gerontológico del buen vivir Mies Guano , realizada en Guano de la provincia de Chimborazo, donde se demuestra que la terapia gestáltica tiene influencia positiva en la elaboración del duelo en adultos mayores, la expresión de emociones hacia el fallecido y cierre de géstales inconclusas, permitió que los duelos retrasados y prolongados que existía en cada uno de los participantes avance y se permita la elaboración total del mismo, restaurando la homeostasis de la psiquis, la aceptación de la realidad de la pérdida y eliminando la sintomatología depresiva reactiva a la pérdida del familiar (Ortiz \& Gaona, 2015).

En esta misma línea, en otra investigación sobre proceso de duelo, mecanismo de afrontamiento y sus estrategias para pérdidas emocionales en personalidades con rasgos dependientes, resalta el papel fundamental del círculo familiar en el desarrollo del sistema de motivos como la resiliencia, autoeficacia, coherencia, desesperanza aprendida, apego y locus de control, teniendo estos mecanismos, la persona tiene esas fortalezas para poder afrontar situaciones emocionales estresantes. El acompañamiento psicológico adecuado permite identificar alternativas $y$ herramientas con las que pueda equilibrar su confianza y ayudar a direccionar el sentido de vida. La ayuda que le otorga la familia en el apoyo emocional es primordial para poder superar el duelo y sus etapas, permitiendo en la persona una vida cómoda junto a su grupo primario de apoyo y consistiendo la pérdida del ser amado (Atiencia,2016).

\section{METODOLOGÍA}

El enfoque metodológico que desarrolla esta investigación es de tipo cualitativo, lo que permitió interpretar y reflexionar respecto a las realidades sociales con la finalidad de hallar el sentido que dan los actores sociales a su realidad.

La presente investigación presenta una metodología de tipo documental y bibliográfica, como menciona 
Hernández y Col (2014) en esta se realiza revisión de material impreso, libros, en la web y otros medios bibliográficos en relación al tema de estudio donde se detallan resultados obtenidos a partir del análisis hermenéutico, posterior a la lectura y comprensión de lo consultado.

Para el desarrollo de este ensayo científico se consideró 26 documentos científicos entre artículos e investigaciones acerca de dinámica familiar y duelo, los cuales fueron buscados en bases de datos como Dialnet, Google académico, Scielo, entre otras. Se eligieron artículos desde el año 1996 hasta el año 2020.

\section{DESARROLLO}

Para profundizar el tema de duelo, es necesario ahondar los conceptos que llevan a este punto, como la muerte, considerada como el final de las funciones vitales del cuerpo, el cese del ciclo vital de las personas, es el destino final de todo ser humano, pese a esto, no se sabe a dónde ni cómo pasará. Por ende, vivir la muerte de un ser amado se consolida como un hecho atroz que llega a marcar la vida de una persona, en este proceso el individuo reluce sus estrategias de afrontamiento ante la pérdida, siendo indispensables para su asimilación (Arias, 2018).

El duelo es un problema de investigación que ha sido considerablemente estudiado por la psicología y sus diversas áreas como el psicoanálisis, la psicoterapia u otras donde se considera que es un problema completo del individuo e involucra un nivel más psíquico. Ciencias como la sociología y la antropología también se encargan de analizarlo investigándolo como un constructo social que asume formas colectivas de realizar durante el duelo y los ritos funerarios en los que se pueden encontrar factores de interés sociológico como: las diferencias socioeconómicas 0 diferencias étnicas y culturales; además estas formas y prácticas de trabajo involucran dimensiones simbólicas que funcionan para ciertos órdenes sociales (Bedoya, 2019).

Si bien, la muerte es una fase natural del ciclo de vida, nadie se encuentra preparado para perder a quienes ama, más cuando sus vínculos afectivos son fuertes, y forman parte de la vida diaria como los miembros 
de la familia, siendo un evento que marca la vida de todo individuo y exige utilizar estrategias de afrontamiento para poder asimilar la pérdida y poder continuar.

Un concepto interesante que ha surgido, es la pedagogía de la muerte, la cual aborda el mecanismo de adaptación a la realidad, donde el individuo es capaz de aprender a aceptar sus propias limitaciones, es así que supera sus miedos y crece interiormente. Se convierte en una clave de orientación de la vida, proporcionando la importancia adecuada a los hechos, siendo más responsable y consciente. Por lo cual la didáctica de la muerte se orienta a enseñar, aprender, desaprender, y reaprender en relación a la autoformación de alumnos y profesores (Gascón, 2007).

La pérdida por muerte implica un ángulo especial por la dificultad del individuo de relatar y ser consciente de la inexistencia de una persona de su realidad. La dificultad se presenta por la imposibilidad de concebir su propia muerte debido a que inconscientemente está convencido de su mortalidad, por esto, se introyecta porque no existe una experiencia de muerte propia para inscribirla en un inconsciente, y las muertes de personas con las cuales crea vínculos estremecen esta idea Freud (1915) citado por (Ramírez, 2017).

El duelo es un proceso que se vivencia tras las pérdidas significativas, se concibe como un conjunto de representaciones mentales y conductas ligadas a pérdidas afectivas, con la finalidad de aceptar la realidad y adaptarse al nuevo contexto (De Hoyos, 2015). Por otro lado, Domínguez (2018) manifiesta que el duelo se considera una respuesta que enmarca la reacción inmediata, que tiene la persona ante el fallecimiento de un ser amado, enfocándose en las posibles consecuencias relacionadas con malestar expresado de diversas formas, estas pueden ser de índole afectivo, fisiológico, emocional y/o presencia de distorsiones cognitivas, entre otras. La intensidad de sintomatología va de acuerdo con el tipo de pérdida, puede ser: ruptura amorosa, pérdida de algún miembro del cuerpo, del trabajo o estatus y por fallecimiento de un ser querido, 
esta última suele vivirse de forma más intensa.

El duelo puede ser interpretado como una experiencia estresante pero normal, debido a que cada individuo tendrá que enfrentar a la pérdida de un objeto que simbolice el valor sentimental o económico, en otras ocasiones se refieren a pérdida por muerte y como esta llega a afectar el desarrollo psicosocial, donde el individuo se plantea qué hacer frente a la situación, es necesario experimentar el dolor, las emociones y sensaciones para evolucionar, y esto dará paso a la autorrealización, finalmente los duelos son parte del crecimiento humano (Bastidas \& Zambrano, 2020).

Ahora bien, en este contexto, la pérdida por muerte lleva un proceso de duelo, que implica una situación universal, cultural, social y personal. La perspectiva antropológica, psicológica y sociológica convergen en la idea de que las personas independientes del contexto sociocultural donde vivan, se apuntan hacia el intento en gran de recuperar aquello que se ha perdido. Como el individuo trata de anteponerse a la pérdida, crea rituales, prácticas funerarias, aparecen mitos, que se expresan de forma simbólica y creativa con la finalidad de comunicar y dar sentido a la muerte (Tizón, 2009).

Ante la muerte de seres amados, siendo este un hecho con connotación cultural, social y personal, en el contexto ecuatoriano, es común que se sigan rituales como prácticas funerarias, vestirse de negro (guardar luto), novenarios y rezos por la liberación de su alma; la familia se enfoca en otorgarle una despedida, evidenciar el aprecio, con la finalidad que su alma descanse. Estos rituales ayudan al sentido de realidad y conciencia de pérdida, cuando no se tiene el cuerpo, es necesario crear rituales para poder iniciar el proceso.

El duelo se expresa de diversas formas, el doliente puede pasar llorando gran parte del día, con imposibilidad de conciliar el sueño, poco apetito, parecer apartado de la realidad, aislamiento, puede haber pensamiento rumiativo de culpabilidad o desesperanza, podría confundirse con la depresión; sin embargo, es normal esta respuesta al inicio de la pérdida por el impacto en su vida, su intensidad y duración, 
puede indicar el desarrollo patológico de la misma.

El proceso de duelo es de índole biopsicosocial, en el cual cada individuo lo vive de forma única, e implica una reorganización de los estados internos y externos que rodean al individuo ocasionando un problema existencial que se comunica en el lenguaje de lo sensible, simbólico y mitológico. En la pérdida, el ritual favorece un espacio que permite proporcionar realidad al hecho, por to cual el cementerio cumple una función más allá del reposo del cuerpo, contribuye a facilitar el proceso (Serna, 2020).

No todo individuo reacciona igual ante la pérdida física de un ser amado, incluso siendo su familia, debido a la individualidad y características personológicas pueden afrontar mejor la pérdida y demostrar sus habilidades de afrontamiento. Cada quien necesita su tiempo, que puede ser distinto al otro, pero es necesario hasta que consiga la reorganización de sus procesos y estados que proporcionan equilibrio.
Cuando el individuo enfrenta la pérdida de alguien amado como un miembro de la familia, empieza un proceso diferenciado que varía y mezcla momentos de duelo donde influye mucho su personalidad, dando espacio a procesos de aislamiento, embotamiento afectivo, retirada esquizoide que se traduce en aislamiento y disociación del duelo, procesos de retirada de la atención y actividad relacionado a lo perdido y aquello que rememora, procesos que mantienen la creencia de reversibilidad de la pérdida, procesos tendentes a reconocimiento de la pérdida, de su relevancia y prevalencia en los vínculos mentales con lo perdido (Parkes, 1998).

Desde un punto de vista constructivista, el duelo se configura como un proceso de recreación de los significados que experimenta una persona después de saber que ha sufrido o perderá. A nivel cognitivo esta transformación es vista como un hecho que continúa en la vida de las personas debido a que la realidad de ganancia y pérdida es en sí misma una experiencia de tiempo necesaria compleja e inevitable. Así la forma en que un individuo responde a la 
pérdida depende de muchos factores incluido el apoyo social, el contexto en el que ocurrió y el tipo de relación con el objeto o sujeto perdido (Chaurand, Feixas, Neimeyer, Salla y Trujillo 2015) citado por (Cala, 2019).

No duele solo la pérdida física, duele la ausencia, las costumbres de lo observado, de las actividades que realizaban juntos, los recuerdos, los buenos momentos incluso lo no resuelto, es aceptar que nunca más se compartirá un espacio en conjunto, por eso el individuo reacciona aislándose físicamente y afectivamente de todos hasta de sí mismo.

El duelo patológico es una de las manifestaciones complejas en este proceso y el sujeto no puede seguir superándolo también se reflejan ciertas reacciones que son inconsistentes con el duelo provocando la mayoría de las consecuencias identificadas como conductas problema que pueden afectar a los individuos a la sociedad en su día a día (Barco \& Vera, 2021). Las incertidumbres y las incógnitas dificultan el proceso de duelo que en última instancia debe desarrollarse a partir de suposiciones y no de juicios sobre la realidad. Luego viene el duelo congelado cuando las experiencias traumáticas son irrefinales y constantemente actualizadas por el sujeto. Esta condición nos imposibilita hacer frente a la pérdida y el proceso de duelo se alarga hasta el infinito prolongando el dolor espiritual (Arbizu, Cepeda \& Kantt, 2020).

Si bien, se ha mencionado al duelo como una reacción natural ante la pérdida, su duración y la persistencia de las características que inciden en la funcionalidad del individuo dan lugar a los duelos complicados, aquellos con altas probabilidades de complicaciones con trabas en las fases que se transforman en un duelo patológico. Tizón (2004) describe tipos de duelos complicados:

- El duelo crónico, aquel cuya duración es extensa y pareciera nunca acabar, siendo consciente que sus esfuerzos no permiten terminarlo, e incapaz de resolverlo por su cuenta.

- El duelo retrasado, suprimido, pospuesto, este es aquel en su inicio se expresó de forma insuficiente, por ende, no se elaboraron las tareas del duelo, 
usualmente ocurre cuando el fallecimiento sucede por suicidios u homicidios, o cuando una pérdida posterior potencia la anormalidad del duelo.

- El duelo exagerado, es aquel donde se encuentra una intensificación mucho más grande de lo esperado, sin embargo, la persona es consciente de que cuadro sintomatológico se vincula con la pérdida y suelen solicitar ayuda. Este duelo patológico, es aquel que desarrolla una depresión, ansiedad, fobias, trastornos de estrés postraumáticos.

- El duelo enmascarado, es aquel en el cual el individuo presenta síntomas que ocasionan adversidades, pero no los relaciona a la pérdida, y suelen asociarlo a otro tipo de problemáticas, usualmente ocurre en niños y adolescentes.

- El duelo desorganizador o bordeline, es aquel que presenta una tendencia a trastornos de estrés postraumático, delirio agudo, crisis confusionales, entre otros.

Por otro lado, referente a los estudios de duelo, se encuentra la tipología de duelos descrita por Varela, Reyes \& García (2017):

El duelo crónico es una situación que también puede estar presente en las pérdidas cuando estos no se resuelven, no elaboran la pérdida del ser amado, presentando resistencia a la aceptación de los hechos, preservando la idea obsesiva de mantenerlo presente, lo cual culmina con paralizar su vida, así su posición de dolor está presente, y no desaparece en el tiempo, perdura por años o no concluye.

El duelo ausente es otra forma de duelo, en esta la persona que ha perdido a alguien importante de su vida, realiza un proceso de bloqueos de sentimientos, ignorando la realidad de los hechos, como si nunca hubiera sucedido, incluso negándolo, evitando la realidad pese al tiempo que ha transcurrido.

El duelo retardado, por su lado presenta similitudes al duelo normal, pero marca una distinción, aunque la persona trate de ignorar el dolor en el inicio, posterior a esto, surge una fuerza a su vez surgen complicaciones las cual debe resolver independientemente. También puede denominarse como 
duelo congelado y surgen personas que controlan sus emociones en exceso y se muestran aparentemente fuertes.

El duelo inhibido, es aquel en el cual hay problemáticas en expresarlo, muy común en la posición de niños, o personas con algún tipo de discapacidad cognitivas, incluso aquellas personas muy tímidas que tienen dificultades de poner su dolor en lenguaje, lo que representa la pérdida, por lo cual, no tienen oportunidades de expresar lo que siente.

El duelo desautorizado, es aquel en que se demuestra un marcado rechazo del contexto social hacia el dolor que experimenta una persona. De esta forma en el paso del tiempo, los demás siempre intentan desautorizar el duelo en algún punto, porque para quien no experimenta el sufrimiento, lo que debe hacer el doliente es dejar ir a quien ya se fue y seguir con su vida

El duelo distorsionado, es aquel que se manifiesta como una fuerte reacción desproporcionada en cuanto a la situación, esto suele ocurrir cuando la persona ya ha experimentado un duelo previo y se encuentra ante una nueva situación de duelo que agrava la situación y lo torna vulnerable.

Cuando un individuo sufre una pérdida significativa como puede ser la muerte de una madre, padre, hermanos, es necesario que sistema familiar este pendiente de los cambios que tenga para afrontar la pérdida, porque no todos logran resolver el duelo, muchos se quedan estancados y atónitos ante ese suceso, por lo cual desarrollan los diferentes tipos de duelos complicados.

El dolor es necesario en el duelo, la capacidad de sentirlo ayuda en el proceso. A nivel intrapsíquico se desarrolla un combate entre la fantasía y la realidad, amor y odio, vida y muerte. Para la resolución del duelo es necesario que el juicio de realidad prevalezca ante la fantasía, el amor ante el odio, y la vida ante la muerte. De esta forma, la posición del psicólogo ocupa un rol significativo en la atención en servicios sociales en general, por su experticia del comportamiento humano, denotando su importancia en la intervención social por la necesidad de incidir sobre el comportamiento de los individuos y colectivo (Arteaga, \& Quinde, 2019). 
Los factores que conciernen al duelo, el tipo de pérdida, el contexto tiene incidencia en la elaboración del duelo, por lo cual, es necesario explorar las características que cruzan al individuo como la identidad y rol de la persona pérdida, edad, sexo, personalidad y situación vital de la persona que sufrió la pérdida, las causas y circunstancias de la pérdida. Además se puede presentar adversidades que compliquen la respuesta emocional del individuo como la muerte súbita, exigencia de cuidados antes de la muerte, como se le informo de la muerte, el manejo de la culpa, muertes de tipo violentas, accidentes o suicidios, también los aspectos psicosociales del que sufre la pérdida como lo es el apoyo y estrés social, las funciones a asumir como el cuidado de personas, la estabilidad económica, reorganización de la vida, y las creencias marcadas en relación a la muerte (Espina \& Pérez, 2005).

El contexto siempre es importante en la resolución de conflictos internos, por lo tanto, es importante en el afrontamiento a la pérdida, cada aspecto incide en el proceso, desde la forma en que se comunica la muerte, el tipo de muerte, y como actúa el sistema tras este hecho, el apoyo que proporcionan, las situaciones pendientes que deja el difunto, todos estos factores influyen significativamente.

Las complicaciones en el duelo se pueden observar mediante las manifestaciones del individuo que se expresan en dependencia afectiva, expresiones de rabia y culpa, vulnerabilidad psíquica previa (antecedentes psicopatológicos y duelos anteriores no resueltos), la falta de control de síntomas durante toda la enfermedad del paciente, problemas económicos, apoyo social, circunstancias de la muerte y contexto cultural (Barreto, 2008).

La muerte de un individuo puede darse por enfermedad $y$ en estas ocasiones pueden culpabilizarse por el cuidado que se le dio al enfermo, o puede ser por accidentes y suelen malinterpretar situaciones con el fin de buscar culpables, y martirizando la homeostasis del sistema, por la vulnerabilidad psíquica de la pérdida puede afectar las relaciones $y$ vínculos de la dinámica familiar.

La muerte es un tema tabú por el dolor que provoca, estos hechos dan como resultado que no se 
aproximara a la profundidad requerida; y que ante la pérdida de un ser querido las personas se sienten enfrentadas a una nueva situación sin una estrategia que les permita afrontar el evento con menos angustia; implica un período posterior de adaptación conocido como duelo (Nestorovic, 2021).

Kubler-Ross (1969) citado por Ávila \& De la Rubia (2013) describía las fases de duelo: negación, ira, pacto/negociación, depresión y aceptación, la cual dura diferentes periodos de tiempo acorde al individuo.

- La negación es la fase en la cual el doliente presenta una imposibilidad de aceptar e identificar la muerte como una realidad.

- La ira es la fase en donde se da una reacción emocional de rabia, envidia, resentimiento y hostilidad por la pérdida, y se lo demuestra en el entorno, a personas que se consideran responsables, médicos, familiares, amistades, Dios, o se dirige a sí mismo, se presenta conductas autodestructivas.

- El pacto/negociación es la fase en que el doliente afronta la culpa, de forma imaginaria o real enfocado en aquellos que dirigió su ira. Se encuentran comprometidos con alcanzar metas terapéuticas de desarrollo personal donde se abandonan las conductas autodestructivas.

- La depresión es la fase donde afloran sentimientos de profundo vacío y dolor ante la pérdida, se torna una actitud de claudicación, muchos suelen dejar el tratamiento en esta fase.

- La aceptación es la fase donde se reconoce la pérdida, el dolor y limitaciones, dejando de victimizar y culpabilizar a otros, asumiendo con responsabilidad los hechos, además se asignan significados a las situaciones que vive.

Las fases del duelo son un proceso que se vivencia ante la pérdida, en la cual cada individuo puede vivir sus propios tiempos e intensidad de forma individual, incluso pueden avanzar una fase y retornar a otra porque no se ha obtenido una conciencia y percepción de realidad que permita continuar plenamente.

Las personas mueren, y su fallecimiento no solo afecta al individuo en particular, esto involucra 
e incide en la familia, por lo cual, es importante conceptualizar esta variable. La familia desempeña un rol en la socialización de los individuos que determina el proceso de desarrollo humano que permite a cada miembro del sistema hacerles frente a las adversidades que se presentan (Hernández, 2009). La familia es un elemento fundamental en la formación de capacidades de sus hijos, y sobre todo en el afrontamiento, que se verá reflejado en las resoluciones de las problemáticas en su vida adulta. No hay que olvidar que la familia es dinámica, flexible por lo cual se percibe como compleja, y las situaciones pueden mejorar 0 empeorar acorde a los cambios que ellos decidan realizar (Palomar, 2012).

La familia como sistema se convierten en un soporte ante las irreparables pérdidas de sus integrantes, sin duda alguna, el cambio de la estructura familiar incide en su dinámica, e invita a una reorganización de roles y funciones que por su flexibilidad podría darse, pero en otras ocasiones se carece de habilidades para afrontar $y$ empeoran el proceso.
La familia es entendida como un lugar de intercambio donde se transmite valores, normas y patrones de comportamiento para moldear el comportamiento de un individuo. Puede estar formada por vínculos de sangre jurídicos sean estos por matrimonio o adopción, por alianzas o pactos tejidos entre sus miembros (Demarchi, Aguirre, Yela \& Viveros, 2016).

La familia puede ser considerada como un sistema que se adapta a los cambios sociales y repercusiones históricas culturales, por lo cual, la familia occidental es un sistema complejo de aprendizaje en interacción. Es así que el entorno familiar representa para un individuo el espacio socio educativo de moldeamiento y aprendizaje de las posibles formas de manejar las situaciones críticas que puede enfrentar en la vida (Amarís, Madariaga y Valle, 2013).

El sistema familiar se integra por personas entrelazados por vínculos sanguíneos o de parentescos, que los relacionan y consolidan como una unidad frente al medio externo, dentro del mismo, es importante definir límites que funcionan como 
líneas de demarcación e intercambio de comunicación, afecto, apoyo, etc., entre dos sistemas o subsistemas. Los límites pueden ser: extrasistémicos, aquellos que delimitan al sistema con el medio externo, e intrasistémicos aquellos que separan y relacionan los distintos subsistemas que forman el sistema más amplio (Bogza,2012).

Cuando una persona fallece, las familias vivencian cambios en su estructura, que en ciertos casos requerirá una intervención que implique una reestructuración, el personal psicológico ayudará a manejar estos aspectos. Los profesionales de salud mental deben tener en cuenta que no existe dos procesos de duelos similares, por lo cual, las familias no experimentarán la pérdida de la misma forma, por ende, es importante considerar al momento de la intervención terapéutica atender las particularidades de las familias, como la cultura, sistema de creencias, el momento centrífugo en el que se sitúan y las disfunciones que pudieran existir (Ceballos, García, 2019).

Vásquez, Nápoles y Sánchez (2004) expresan sobre la conceptualización de la dinámica familiar, que se puede entender como una colección de fuerzas positivas y negativas que trascienden en el comportamiento de cada miembro de la familia consiguiendo que esta unidad, funcione bien o mal. La dinámica familiar permite vislumbrar a esta primera institución, la cual ejerce su influencia desde la infancia de cada miembro por medio de la transmisión de valores, costumbres y creencias por medio de la convivencia diaria; siendo esta, la primera institución educativa y socializadora del niño (Guevara, 1996).

En los estudios realizados sobre las etapas del duelo familiar, se han encontrado datos importantes que permiten comprender de mejor manera la repercusión en el sistema familiar. En el aspecto comunicacional se detallan aumento o disminución en la comunicación a nivel general o en temas concretos, los patrones comunicacionales presentan una variación, además de cambios en los canales. A nivel estructural suele existir confusión en la jerarquía familiar, en la redistribución de las alianzas familiares, en los roles asignados. También se ven afectadas las 
relaciones con el medio externo que se identifica con el aislamiento de los miembros del sistema, el rechazo a los soportes o redes de apoyo o con sobreprotección de los miembros (Moos, 1995) citado por (Bogza, 2012).

En las complejidades del proceso familiar de duelo por suicidio se identifican ciertos componentes que pueden servir de guías tanto en la comprensión como en las intervenciones terapéuticas con estas familias. Nos referimos al tiempo de evolución familiar como es el ciclo vital y quién realiza la acción la presencia 0 ausencia de un mensaje de rechazo y explicación la forma y ubicación de la acción el patrón de comunicación la sucesión de la familia anterior la relación entre cada persona perdida, de cada miembro de la familia y de toda la familia y la voluntad de apoyar y acompañar no sólo a las familias de origen sino también a los grupos locales más cercanos emocionalmente. Estos definirán y marcarán entre otros factores cómo el funcionamiento de la familia construye el comportamiento y por tanto su desarrollo y evolución (San Martín, 2019).
El sistema familiar sufre por la pérdida de sus miembros, por el lugar o posición que ocupaba, lo que brindaba en los diferentes holones. Se encuentra variaciones que requerirán una reacomodación, en el nivel comunicacional puede oscilar entre un aumento o disminución de temas que retornan en la muerte, o pueden vincular a estrategias para continuar, es común que las jerarquías se vean afectado y se encuentren desconcertados de que hacer, quien asume las funciones del faltante, y muchas veces se aíslan del mundo, rechazan ayuda, o se orientan a una sobreprotección por miedo a una nueva pérdida.

Tras la pérdida de un miembro, la familia tratará de reasignar roles, los integrantes de la estructura familiar suplen el rol debido a que se siente un temor a la desorganización como creencia relacional. Cuando al fallecido se lo sigue nombrando como punto de referencia, sigue vivo. En este sentido, el terror a la aceptación está presente, se pasa por una desorganización angustiante lo que dificulta avanzar en su proceso (Concha \& Hernández, 2008). 
Los síntomas se pueden identificar en el sistema con persistencia e intensidad en alguno de sus miembros, que llega a detener su vida orgánica, familiar, académica, relacional, laboral, dando lugar a un duelo patológico, siendo un indicador de necesidad de atención psicológica como parte del proceso. Sin duda alguna, la pérdida modifica la estructura familiar y necesita de una reorganización del sistema familiar total (Concha \& Hernández, 2008).

Las pérdidas por muerte pueden ser inminentes 0 anticipadas, la diferencia radica en la preparación psicológica que adquieren los miembros del sistema familiar en relación a vivir con la ausencia de la persona fallecida que hasta ese entonces formaba parte de su vida, suponen una desestructuración del sistema familiar. Tanto en los casos que la muerte es inminente o que la persona se encuentra en fase terminal y su pérdida es inevitable inciden en lo intimo de la afectividad de los dolientes, esta afectación crea incertidumbre en el sistema familiar (Rolland, 2000) citado por (Torres, 2019).
El dolor y el sufrimiento obtenido como consecuencia del fallecimiento entristece a la trama de las interacciones de los integrantes de la familia, posteriormente involucran las relaciones que conservan con conocidos, incluso se da el mismo caso en las personas que no supieron nada acerca del difunto (Tenecela, 2020). El sistema familiar y su dinámica puede salir tan afectada que afectará la vida de cada miembro porque sienten que su vida se detuvo, y fallando en las diferentes áreas en que desenvuelve, siendo este un indicador de una patología, ya deja de ser un proceso de duelo común y necesitará intervención psicológica para que logren reorganizarse.

\section{CONCLUSIONES}

El duelo es un proceso natural ante la pérdida de personas significativas en la vida de cualquier individuo, en este periodo la persona deberá afrontar las adversidades ante la ausencia de la persona y en su sistema familiar deberán adaptarse y conseguir una reorganización para poder continuar y evitar estancarse, la dinámica se verá afectada si los miembros pierden su capacidad de 
expresar su sentir, más cuando su comunicación se centra en reclamos, culpabilización, y deteriora los vínculos, por lo cual puede desarrollarse los diferentes tipos de duelo complicado.

El duelo complicado incide significativamente en la funcionalidad del individuo, como este se muestra ante los demás, el cumplimiento de su rol y funciones, por lo cual, incide en la relación que establece con personas de su círculo cercano como es la familia. Cuando el individuo eleva sus barreras evitando comunicarse con el otro, llegara un momento en que la tensión acumulada se manifieste y afecte los elementos de la dinámica familiar.

\section{BIBLIOGRAFÍA}

Amarís, M., Madariaga, C. y Valle, M. (2013). Estrategias de afrontamiento individual $y$ familiar frente a situaciones de estrés psicológico. Revista Psicología desde el Caribe, 30(1), 123-146.

Arbizu, J., Cepeda, C., \& Kantt, M. (2020). Duelo sin cuerpo. Experiencias con familiares de desaparecidos en el mar. Anuario de Investigaciones de la Facultad de Psicología, 5(7), 141-147. Disponible en https://revistas.unc.edu.ar/ind ex.php/aifp/article/view/31684 /32522

Arias Sempértegui, M. I. (2018). Estrategias de afrontamiento frente al duelo por la muerte de un ser querido en estudiantes de 18 a 25 años en una universidad de Quito (Bachelor's thesis, Quito: Universidad de las Américas, 2018).

Arteaga, E. M. B., \& Quinde, O. R. Z. (2019). El trabajo fisioterapéutico y las etapas del duelo: Un enfoque interdisciplinario. Dominio de las Ciencias, 5(2), 44-55.

Atiencia Heredia, J. P. (2016). Proceso de duelo: un mecanismo de afrontamiento $y$ sus estrategias para pérdidas emocionales en personalidades con rasgos dependientes. Recuperado del sitio web http://186.3.32.121/bitstream/ 48000/8019/1/T1893_ATIENCIA\%20HEREDI A\%20JUAN\%20PABLO.pdf

Ávila, M. M., \& de la Rubia, J. M. (2013). El significado psicológico de las cinco fases del duelo propuestas por Kübler-Ross mediante las redes semánticas naturales. Psicooncología, 10(1), 109130. Recuperado del sitio web 
https://core.ac.uk/download/p df/38820309.pdf

Barco Soledispa, J. J., \& Vera Cáceres, L. A. (2021). Estrategias de Afrontamiento ante el Duelo por pérdida de Familiares durante la Pandemia por COVID-19 (Bachelor's thesis, Universidad de GuayaquilFacultad de Ciencias Psicológicas). Disponible en http://repositorio.ug.edu.ec/ha ndle/redug/56762

Barreto, P., Yi, P., \& Soler, C. (2008). Predictores de duelo complicado. Psicooncología, 5(2), $383-400$.

Bastidas-Martínez, C. J., \& Zambrano-Santos, R. O. (2020). El duelo familiar y el desarrollo emocional en los estudiantes. Revista Arbitrada Interdisciplinaria Koinonía, 5(9), 125-144. Disponible en https://www.redalyc.org/journ al/5768/576869060007/5768 69060007.pdf

Bedoya Ramírez, J. C. (2019). "Entre el duelo y el afrontamiento" recomposición familiar $y$ personal tras la muerte del padre (Doctoral dissertation). Disponible en

Bogza, I. A. (2012). La terapia familiar sistémica y el duelo.

Cala Aparicio, W. L. (2019). Recursos y capacidades resilientes en familias ante duelo por muerte parental. Disponible https://repository.upb.edu.co/ bitstream/handle/20.500.1191 2/6379/digital_38690.pdf?seq uence $=1$ \&isAllowed $=y$

Cely Araque, E., Martínez Eljadue, M. A., \& McLeod Muñoz, A. L (2016). Transformaciones en la dinámica familiar causadas por el deceso de un familiar. Recuperado del sitio web https://repository.javeriana.ed u.co/handle/10554/36320

Ceballos, G., Vásquez, G., Nápoles, R. y Sánchez, T. (2004). Influencia de la dinámica familiar $y$ otros factores asociados al déficit en el estado nutricio de preescolares en guarderías del Sistema para el Desarrollo Integral de la Familia (DIF) Jalisco, Hospital Civil de Guadalajara e Instituto de Nutrición Humana. Guadalajara (México): Universidad de Guadalajara.

Concha Santander, D., \& Hernández Briceño, J. (2008). Duelo parental y cambios en el sistema familiar: padres en duelo, Corporación Renacer, comuna La Florida (Doctoral dissertation, Universidad Academia de Humanismo Cristiano). Recuperado del sitio web http://bibliotecadigital.academ ia.cl/xmlui/bitstream/handle/1 23456789/1607/ttraso244.pdf ?sequence $=1$ \&isAllowed $=y$

Demarchi Sánchez, G., Aguirre Londoño, M., Yela Lozano, N., 
\& Viveros Chavarria, E. (2016). Sobre la dinámica familiar. Revisión documental. Cultura educación y sociedad. Recuperado de https://repositorio.cuc.edu.co/ bitstream/handle/11323/3107/ SobRe\%20la\%20din\%c3\%a1 mica\%20familiaR.\%20ReviSi \%c3\%b3n\%20documental.pd $\mathrm{f}$ ? sequence $=1$ \&isAllowed $=\mathrm{y}$

Domínguez Mercado, D. L. (2018). Duelo complicado por suicidio(Doctoral dissertation, Universidad Autónoma de Aguascalientes)

Espina, A., Gago, J., \& Pérez, M. (2005). Sobre la elaboración del duelo en terapia familiar. Revista de psicoterapia, 4(13), 77-87. Recuperado del sitio web http://www.centrodepsicotera pia.es/pdf_art/22-

Sobre\%20la\%20elaboracion \%20del\%20duelo\%20en\%20t erapia\%20familiar.pdf

Gaona Pardo, P. A., \& Ortiz Guadalupe, M. O. (2015). Terapia gestáltica en la elaboración del duelo en adultos mayores no institucionalizados del centro gerontológico del buen vivir mies-guano, período noviembre 2014-abril 2015 (Bachelor's thesis, Riobamba: Universidad Nacional de Chimborazo, 2015.). recuperado del sitio web http://dspace.unach.edu.ec/h andle/51000/1016
García Torres, R. (2019). El proceso de duelo: una revisión desde la Terapia Familiar Sistémica y la Teoría del apego. Disponible en https://repositorio.comillas.ed u/xmlui/handle/11531/33587

Gascón, A. de la H. (2007). Fundamentos para una Pedagogía de la Muerte. Revista Iberoamericana de Educación, 41(Extra 2), 5.

Guevara N., G. (1996). La relación familia-escuela. Educación 2001, 9, 6-13.

Hernández, H. (2009). Familia, ciclo vital y psicoterapia sistémica breve. Bogotá, Colombia: Editorial el Búho.

Hernandezi, R., Fernández, C., \& Bautista, P. (2014). Metodología de la Investigación. México: Mcgraw-hill/Interamericana Editores. S.A. DE C.V.

De Hoyos López, M. C. (2015). ¿Entendemos los adultos el duelo de los niños? Acta Pediátr Esp [revista en internet], 73(2), 27-32. Recuperado del sitio web https://www.neurologianeonat al.org/wpcontent/uploads/2018/05/ENT ENDEMOS-LOS-ADULTOSEL-DUELO-NIN\%CC\%83OSACTA-PEDIATRICA.pdf

Martínez, C. J. B., \& Santos, R. O. Z. (2020). El duelo familiar y el desarrollo emocional en los 
estudiantes. Revista Arbitrada Interdisciplinaria koinonia, 5(9), 125-143. Recuperado del sitio web https://dialnet.unirioja.es/servl et/articulo?codigo $=7220305$

Nestorovic Monsalve, D. M. (2021). Estrategias de afrontamiento ante el duelo por pérdida de un miembro familiar. Disponible https://repositorio.ulima.edu.p e/bitstream/handle/20.500.12 724/13221/Nestorovic_Monsa Ive. pdf?sequence $=1$ \&isAllow ed $=y$

Parkes, C.M. (1998): Traditional Models and Theories of Grief. Bereavement Care, 17, (2), 21-23.

Palomar, G. (2012). Estudio de las estrategias de afrontamiento en adolescentes y su relación con el funcionamiento familiar $y$ el apoyo social (Tesis Doctoral inédita, Universidad de Oviedo, España). Recuperada de www.redalyc.org/pdf/213/213 28600007.pdf

Ramírez, R. D. P. G. (2017). Proceso de duelo: meta análisis del enfrentamiento a la muerte suicida desde una perspectiva familiar.

Cuadernos

Hispanoamericanos de Psicología, 17(1), 49-64. Disponible en https://revistas.unbosque.edu .co/index.php/CHP/article/vie $\mathrm{w} / 2147 / 1617$
San Martín, J. M. A. (2019). Suicidios y Familias. Ingredientes en la evolución de los procesos de duelo y líneas de intervención. Revista REDES, (39). Disponible en http://redesdigital.com.mx/ind ex.php/redes/article/view/281/ 6783455

Tenecela Mainato, A. S. (2020). Depresión por duelo y su influencia en el núcleo familiar de un adulto (Bachelor's thesis, Babahoyo: UTB, 2020). Disponible en http://dspace.utb.edu.ec/bitstr eam/handle/49000/9027/EUTB-FCJSE-PSCLIN000407.pdf?sequence $=1$ \&is A llowed $=\mathrm{y}$

Tizón, J. L. (2009). Perdida, pena, duelo: vivencias, investigación y asistencia. España: Paidós.

Torres, R. G. (2019). El proceso de duelo desde la Terapia Familiar Sistémica: el papel del apego adulto. Miscelánea Comillas. Revista de Ciencias Humanas y Sociales, 77(151), 341-363.

Varela López, L. L., Reyes Monroy, C. A., \& García, J. (2017). Tipos de duelo y estrategias de afrontamiento. Recuperado del sitio web https://repository.ucc.edu.co/ handle/20.500.12494/14552 